\title{
Modelling Multi-Frequency Pol-InSAR Data from the Percolation Zone of the Greenland Ice Sheet
}

\author{
Georg Fischer, Student Member, IEEE, Konstantinos P. Papathanassiou, Fellow, IEEE, and Irena \\ Hajnsek, Fellow, IEEE
}

\begin{abstract}
The analysis of data from an airborne synthetic aperture radar (SAR) campaign in the percolation zone of Greenland revealed an interferometric coherence undulation behavior with respect to vertical wavenumber, which cannot be explained with existing models. We propose a model extension that accounts for scattering from distinct layers below the surface. Simulations show that the periodicity of the coherence undulation is mainly driven by the vertical distance between dominant subsurface layers, while the amplitude of the undulation is determined by the ratio between scattering from distinct layers and scattering from the firn volume. We use the model to interpret quad-pol SAR data at $\mathrm{X}$-, C-, S-, L-, and P-band. The inferred layer depths match layer detections in ground based radar data and in situ measurements. We conclude that in the percolation zone scattering from subsurface layers has to be taken into account to correctly interpret SAR data and demonstrate the potential to retrieve geophysical information about the vertical subsurface structure.
\end{abstract}

Index Terms-Glacier, ice sheet, polarimetric SAR interferometry (Pol-InSAR), stratigraphy, synthetic aperture radar (SAR), vertical structure.

\section{INTRODUCTION}

$\mathrm{T}$ he dynamics of glaciers and ice sheets are an important indicator of climate change with implications on environment and society [1]. While mass balance estimations are derived today from remote sensing data, uncertainties due to subsurface structure and density related to melt-refreeze processes remain [1][2]. Synthetic Aperture Radar (SAR) measurements provide a way to address these uncertainties due to their penetration into glaciers and ice sheets, especially at lower frequencies, and their sensitivity to different conditions of snow, firn and ice. This potentially allows inferring

Manuscript submitted for review December 22, 2017. This work is supported by the Initiative and Networking Fund of the Helmholtz Association, in the frame of the 'Helmholtz Alliance Remote Sensing and Earth System Dynamics'.

G. Fischer and I. Hajnsek are with the Polarimetric SAR Interferometry Research Group, Microwaves and Radar Institute (HR), German Aerospace Center (DLR), 82234 Wessling, Germany, and also with the Swiss Federal Institute of Technology (ETH) in Zurich, 8092 Zurich, Switzerland (e-mail: georg.fischer@dlr.de; irena.hajnsek@dlr.de).

K. P. Papathanassiou is with the Information Retrieval Group, Microwaves and Radar Institute (HR), German Aerospace Center (DLR), 82230 Wessling, Germany (e-mail: kostas.papathanassiou@dlr.de). information about the vertical subsurface structure. However, the methodology and the algorithms to extract physical vertical structure parameters from SAR data are not established, because it is not yet fully understood how the vertical structure below the surface affects SAR measurements.

Early studies make use of SAR backscatter coefficients to estimate accumulation rates [3][4] and to map glacier zones [5]. One step forward towards subsurface characterization was the development of decompositions and models to interpret polarimetric SAR (PolSAR) data from glaciers and ice sheets. Based on polarimetric studies that indicated the sensitivity of polarimetric backscatter to glacier zones [6][7], first polarimetric models considered ice lenses and glands as the main scattering contributions [8]. Further advances in polarimetric modelling improved the ability to describe the subsurface of an ice cap in Svalbard [9][10]. However, PolSAR measurements cannot resolve the vertical distribution of the scattering contributions.

The vertical distribution of scatterers in the subsurface can be retrieved by SAR interferometry (InSAR). Hoen and Zebker [11] modelled the subsurface of ice sheets as a uniform lossy volume with constant extinction and infinite depth to analyze InSAR coherence measurements at C-band. This approach allows the estimation of the penetration depth of InSAR signals which is related to the vertical structure of scatterers. The dependency of the penetration depth on different snow, firn and ice conditions was also reported by Rignot et al. [12]. A model proposed by Oveisgharan and Zebker [13] aimed to relate accumulation rates in the dry snow zone of Greenland to InSAR coherences by assuming a layered firn body. Even if the study was hampered by a time difference of 83 years between the SAR measurements and validation data, it indicated the potential to analyze vertically layered firn with InSAR. Nevertheless, the uniform volume assumption [11] is still used in recent studies for the characterization of ice sheets [14].

The combination of both aforementioned techniques, by means of polarimetric SAR interferometry (Pol-InSAR) [15], allows to investigate the vertical distribution of different scattering processes and was successfully used to estimate physical structure parameters from natural volumes, particularly in forest applications. Few studies have assessed the potential of Pol-InSAR to retrieve geophysical information from glaciers and ice sheets. Sharma et al. [16] employed a 
polarimetric decomposition to separate surface from volume scattering contributions and then used the uniform volume model [11] to retrieve extinction coefficients at L- and P-band over the Austfonna ice cap in Svalbard. Stebler et al. [17] demonstrated that different subsurface structures at different glacier zones have a clear separable effect on Pol-InSAR coherences. Despite this, the existing approaches are still very limited in the interpretation of Pol-InSAR data [18][19] and therefore also in the retrieval of geophysical information, since the combination of a uniform volume and scattering from the surface has only limited flexibility to represent different scattering scenarios from glaciers and ice sheets.

Recent polarimetric studies improved the understanding of anisotropic propagation behavior of snow [20] and firn [10], and investigated the polarimetric scattering characteristics from oriented particles within the firn volume [10]. This overcomes assumptions made in earlier Pol-InSAR studies [16] about scattering from sastrugi, which is only applicable to specific areas if acquired under certain radar aspect angles.

New developments indicated the necessity to consider the impact of subsurface layers on InSAR coherence [21][22]. The term layer is used in literature both for thin layers associated with surface scattering mechanisms [13][16][20][22] as well as extended firn bodies associated with volume scattering $[10][11][13][16][19]$. We use the first meaning, referring to clear discontinuities within the firn column in the percolation zone of glaciers and ice sheets. We refer to the first layer as the snow-firn interface, which corresponds to the surface at the end of the last summer, ignoring a shallow winter snow pack, which is likely transparent at microwave frequencies if it is dry and did not experience melting [20].

Even though Hoen [23] and Oveisgharan and Zebker [13] already considered a layered subsurface to improve the correlation between modelled and in situ accumulation rates, a direct assessment of subsurface layer effects in InSAR data is missing. A concise modelling effort of such effects, with limited a priori assumptions, may facilitate the development of robust retrieval algorithms.

The objective of this paper is to advance these findings and to propose a realistic scattering model for the interpretation of Pol-InSAR data collected in the percolation zone of Greenland.

In Section II the experimental SAR data and ground measurements of this study are described. The proposed multiple-layer-plus-volume model is introduced and discussed in Section III. In Section IV the SAR data are analyzed with respect to the model characteristics, followed by discussions and conclusions in Section V.

\section{EXPERIMENTAL DATA}

\section{A. ARCTIC15 Campaign and the South Dome Test Site}

This study is based on an airborne SAR data set acquired during April and May 2015 in Greenland with DLR's F-SAR system [24] in the frame of the ARCTIC15 campaign. Fully polarimetric, multi-baseline interferometric SAR data were acquired at five different frequencies (X-, C-, S-, L-, and

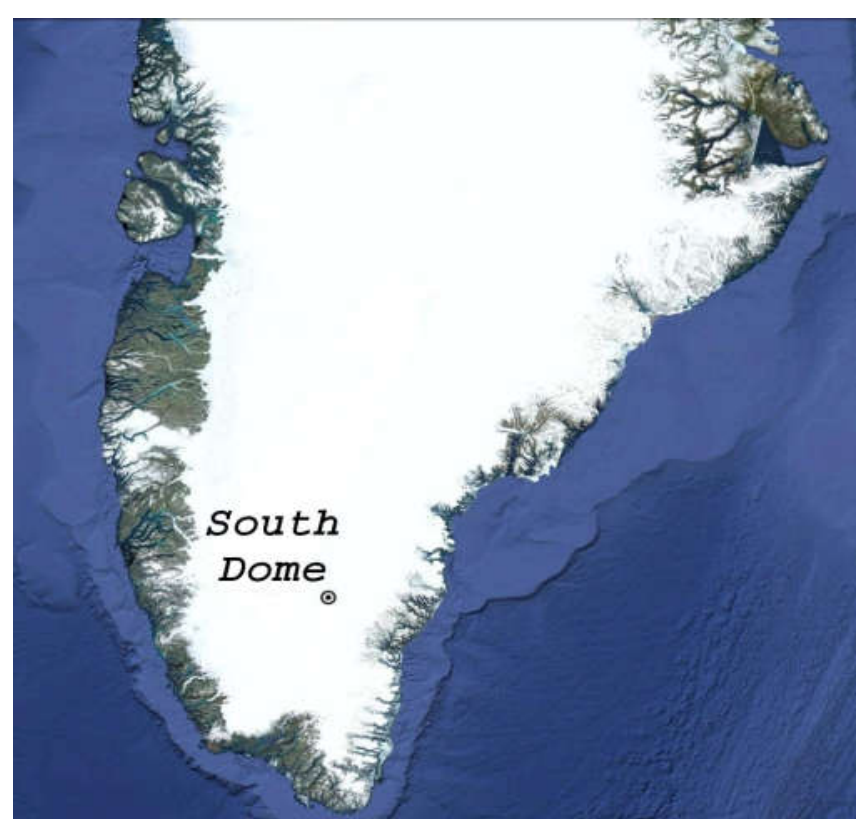

Fig. 1. Location of the South Dome test site in the southern part of Greenland.

P-band; 9.6 GHz, 5.3 GHz, 3.3 GHz, $1.3 \mathrm{GHz}$, and $0.44 \mathrm{GHz}$, respectively) over different glacier zones.

This study focuses on the South Dome test site $\left(63.52^{\circ} \mathrm{N}\right.$, $44.54^{\circ} \mathrm{W}$, Alt.: $2868 \mathrm{~m}$, see Fig. 1), where SAR data covering an area of approximately $6 \mathrm{~km} \mathrm{x} 3 \mathrm{~km}$ were acquired. Coordinated ground activities were carried out by DLR and ETH Zurich, which comprised the placement of corner reflectors, the acquisition of ground penetrating radar (GPR) and GPS profiles as well as manual probing of near surface layers with an accumulation probe.

The F-SAR was flown at $3000 \mathrm{~m}$ altitude above ground, with six to eight parallel flight tracks from two opposite headings. The nominal horizontal baselines were 10-90 m for the X-, S-, and L-band acquisitions and 5-35 $\mathrm{m}$ at the opposite heading at $\mathrm{X}-, \mathrm{C}-$, and L-band. P-band data was acquired during a separate flight 16 days later with nominal horizontal baselines of 10-270 $\mathrm{m}$. The incidence angle varies from $25^{\circ}$ to $60^{\circ}$ and the spatial resolution is between $0.5 \mathrm{~m}$ (azimuth) $\times 0.6 \mathrm{~m}$ (slant range) at $\mathrm{X}$-band and $1.0 \mathrm{~m} \times 3.8 \mathrm{~m}$ at P-band.

The different baselines and the incidence angle variation lead to a wide range of vertical wavenumbers $k_{z}$ that has been exploited in this study. In all frequencies $k_{z}$ values below $0.1 \mathrm{rad} / \mathrm{m}$ exist, except for S-band where the smallest value is $0.2 \mathrm{rad} / \mathrm{m}$. The maximum $k_{z}$ values are at least $2.8 \mathrm{rad} / \mathrm{m}$ in all frequencies.

Temporal decorrelation can be neglected for the ARCTIC15 data with only about 15 min between consecutive acquisitions and a maximum temporal separation of $1 \mathrm{~h} 45 \mathrm{~min}$ at stable negative temperatures.

Noise decorrelation $\gamma_{S N R}$ is well above 0.94 (above 0.99 at L- and P-band), due to the strong backscatter in the percolation zone of Greenland. Therefore, its effect on the presented results is marginal. Nevertheless, the magnitude of the interferometric coherence $|\gamma|$, which this paper focuses on, is corrected for $\gamma_{S N R}$ by means of the polarimetric HV-VH coherence [25]. 


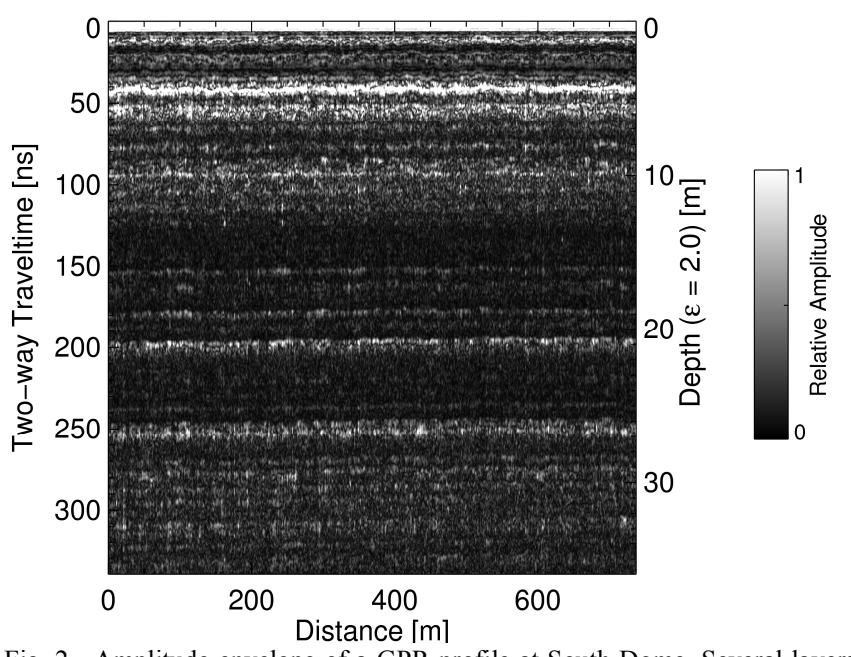

Fig. 2. Amplitude envelope of a GPR profile at South Dome. Several layers with varying backscattered power are visible.

Each interferometric image pair was common bandwidth filtered individually, accounting for the variation of the incidence angle over range [26].

The coregistration was performed using a reference DEM generated from X-band single-pass InSAR data acquired by F-SAR during the campaign.

\section{B. Ground Measurements}

South Dome is located in the percolation zone, where refreezing of seasonal melt water leads to high density ice layers in the firn column, which are considered strong scatterers in both GPR and SAR data. Therefore, GPR data can provide the necessary information on the vertical subsurface structure to understand its influence on Pol-InSAR measurements. In particular, layers related to years with stronger melting are visible in GPR profiles, which is confirmed by firn cores [27].

For this purpose, more than $2 \mathrm{~km}$ of GPR transects were acquired one week before the X-, C-, S-, and L-band and two weeks after the P-band acquisitions using a $500 \mathrm{MHz}$ pulse radar system. The profiles acquired before and after the SAR acquisitions are very similar without any recognizable differences.

The time window of the GPR measurements was $377 \mathrm{~ns}$, corresponding to a depth range of almost $40 \mathrm{~m}$, even though the last $5 \mathrm{~m}$ are dominated by noise. The theoretical vertical resolution is about $20 \mathrm{~cm}$ in firn. A basic processing chain was applied, including removal of direct current bias, time-zero correction, bandpass filtering and gain adjustment [28].

One example of our GPR measurements at South Dome is shown in Fig. 2, revealing the aforementioned stratigraphy with its distinct layers and a particular strong one at about $-4.5 \mathrm{~m}$ depth.

The layered subsurface structure visible in our GPR data was confirmed by echograms from the Accumulation Radar instrument of NASA's Operation IceBridge [29] acquired in April 2014. The effect of the one year difference is limited due to the average accumulation rate of approximately $0.5 \mathrm{~m}$ water equivalent per year [30]. The large coverage of Accumulation
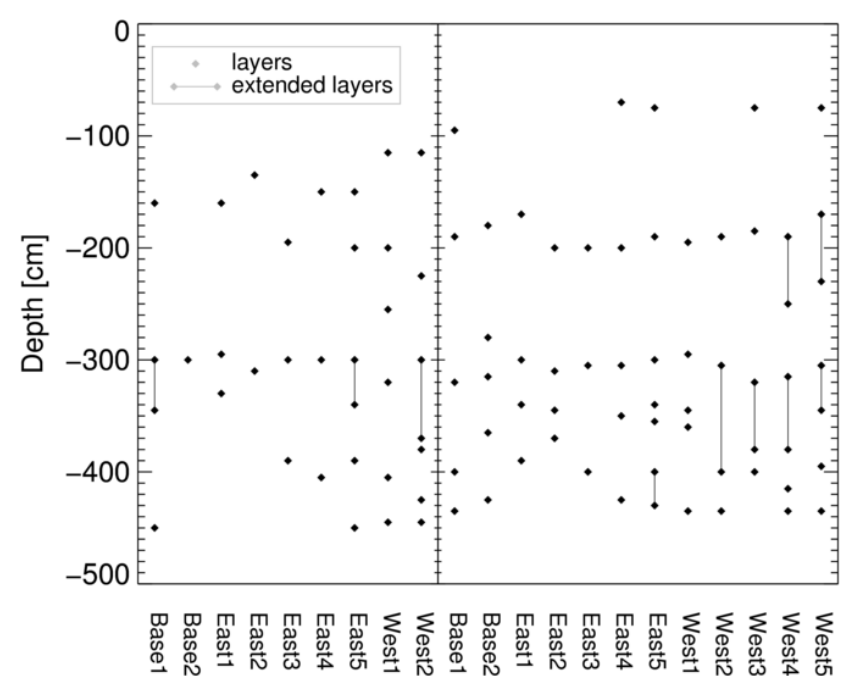

Fig. 3. Manual layer probing results at South Dome from measurements before (25 April 2015, left part) and after (28 May 2015, right part) the SAR acquisitions.

Radar data confirmed the horizontal homogeneity of the subsurface structure in the South Dome area at larger distances then our SAR coverage. Therefore, we consider the GPR profiles to be representative for the entire test site.

Complementary information about stratigraphy in the first 3-4 m was collected with an accumulation probe, which was used to manually detect hard layers within the firn. The layer detections for South Dome are plotted in Fig. 3. Two to five samples were taken at three locations in the SAR scene center before and after the SAR acquisitions. The consistency of the layer readings was encouraging even though the individual point measurements have a randomness to hit or miss ice lenses that form the layers. The depth accuracy of the manual readings on the accumulation probe was assessed to be $\pm 5 \mathrm{~cm}$, which is accurate enough for our purposes. These measurements are considered reliable only in case of consistently detected layers. Thinner and weaker layers were likely to be penetrated without a clear reading, e.g. the inconsistent detections around $-1 \mathrm{~m}$ depth in Fig. 3.

The layer at $-3 \mathrm{~m}$ was detected most consistently, with further detections just a few centimeters below which were sometimes recorded as a single thicker layer. Based on the average accumulation rate [30] and Snow Radar data [31], the spacing of annual layers is about $1 \mathrm{~m}$, which matches our manual measurements, even though potential layers at $-1 \mathrm{~m}$ and $-2 \mathrm{~m}$ were not consistently detected.

All our in situ and SAR data were acquired within 33 days. We consider the situation temporally stable throughout this period with negative temperatures and only $1 \mathrm{~cm}$ snow height change based on data from the South Dome weather station of the Greenland Climate Network [32].

\section{PolSAR Assessment}

South Dome is the highest elevated area in the south of Greenland and is a flat and horizontally homogeneous plateau with a maximum elevation difference of less than $20 \mathrm{~m}$ within 

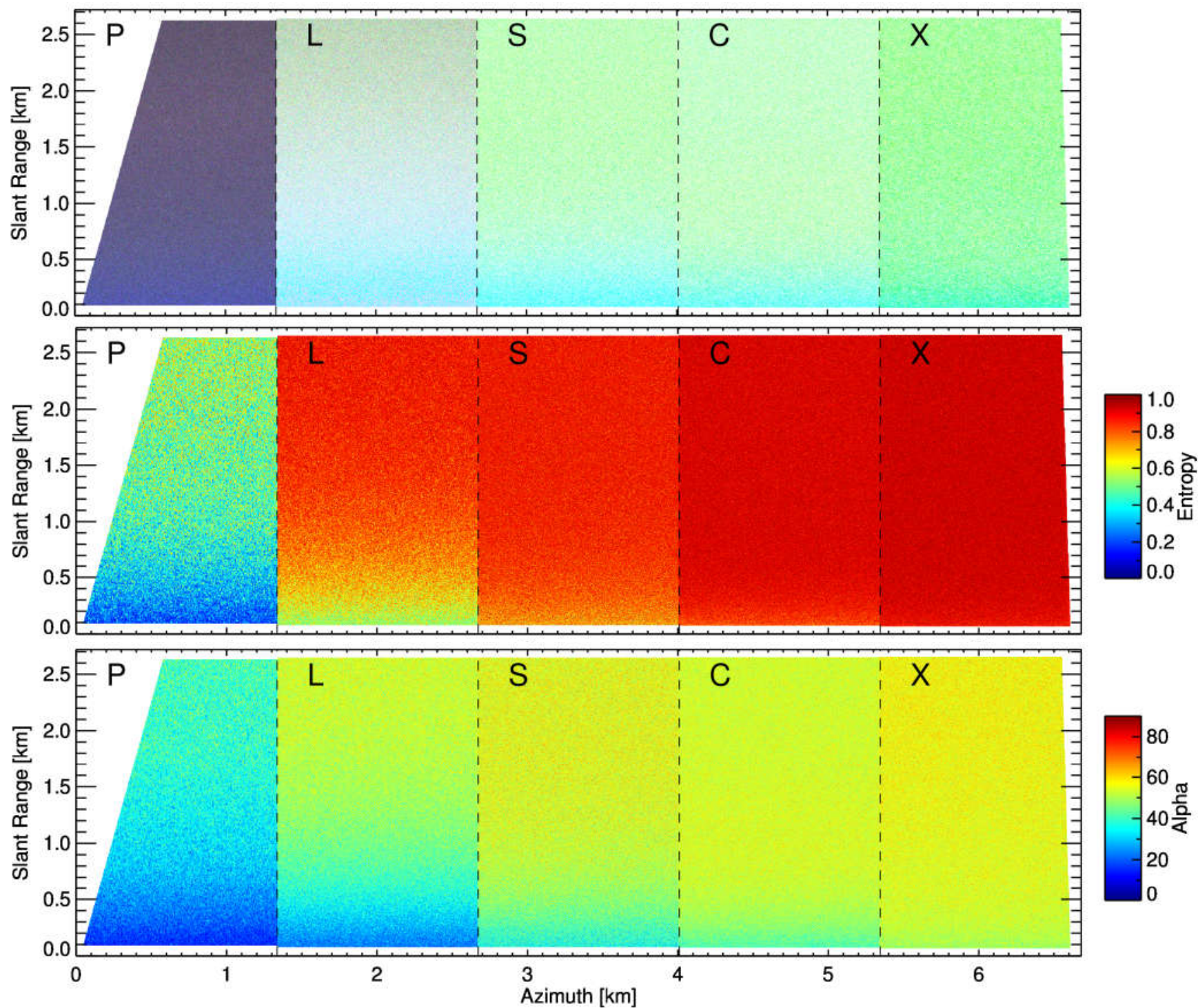

Fig. 4. Top: Multi-frequency Pauli representation (HH+VV: blue, HH-VV: red, HV: green) of the South Dome test site at $63.52^{\circ} \mathrm{N}, 44.54^{\circ} \mathrm{W}$, Alt.: $2868 \mathrm{~m}$. Middle: Scattering entropy parameter. Bottom: Scattering alpha parameter. Due to the horizontal homogeneity of the area, P-, L-, S-, C-, and X-band are aggregated into a single graphic for illustration purposes. Within one frequency, only a range trend due to incidence angle variation is present, while the azimuth dimension is constant across the entire scene.

the area imaged by the SAR. This homogeneity is also revealed by the Pauli color composite images of polarimetric backscatter in Fig. 4 (top). Since there are no visible changes along azimuth for any given frequency, we aggregated all five frequencies into a single image. Within one frequency, only a range trend is present due to the incidence angle variation indicating stronger scattering in $\mathrm{HH}+\mathrm{VV}$, typically interpreted as surface scattering, in near range. The overall very strong HV channel, which is increasing with frequency, is typically associated with volume scattering.

Conventional polarimetric analyses by means of the entropy and alpha scattering parameters [33] indicate dominant volume scattering with overall high entropy values, e.g. above 0.8 , and alpha angles between $40-60^{\circ}$ at X-, C-, S-, and L-band. At $\mathrm{P}$-band entropy ranges between $0.2-0.55$ and alpha between $15-40^{\circ}$. Scattering entropy and alpha for all frequencies are shown in Fig. 4 (middle and bottom). An increase with incidence angle can be observed, and entropy and alpha saturate around 0.9 and $55^{\circ}$, respectively, for X-, C-, S-, and L-band. This increase of both parameters with incidence angle is related to the fact that volume scattering decreases significantly less (theoretically not at all) with incidence angle than surface scattering. At the same time, entropy and alpha increase with frequency due to an increased depolarization of surface scattering induced by the increase in effective roughness. Also, smaller particles may contribute additionally to volume scattering at higher frequencies.

Backscatter values are well above $-15 \mathrm{~dB}$ even at $\mathrm{HV}$ at $60^{\circ}$ incidence angle for L-band and increase up to $0.7 \mathrm{~dB}$ at $28^{\circ}$ incidence angle in $\mathrm{HH}$. Only at P-band $\mathrm{HV}$ drops to $-29 \mathrm{~dB}$. The noise contribution to the high entropy values is marginal, since the signal-to-noise ratio stays above $11 \mathrm{~dB}$ for all frequencies and polarizations. 


\section{InSAR Coherence Profiles at L-band}

While the polarimetric backscatter analysis indicated only a frequency and incidence angle dependence, the interferometric analysis revealed an "unusual" undulation pattern of the InSAR coherence [22], which was the initial trigger of this study.

The complex interferometric coherence $\gamma$ is obtained by forming the normalized cross-correlation between the interferometric image pair $s_{1}$ and $s_{2}$ at a given polarization $\vec{w}$ $[15][33]$

$$
\gamma(\vec{w})=\frac{\left\langle s_{1}(\vec{w}) s_{2}^{*}(\vec{w})\right\rangle}{\sqrt{\left\langle s_{1}(\vec{w}) s_{1}^{*}(\vec{w})\right\rangle\left\langle s_{2}(\vec{w}) s_{2}^{*}(\vec{w})\right\rangle}}
$$

After common bandwidth filtering, the compensation of additive noise decorrelation and neglecting temporal decorrelation, the remaining volume coherence depends on the vertical distribution of backscattering $\sigma_{v}(z)$, stretching from $z_{0}$ at the glacier surface (i.e. snow-firn interface, respectively the surface at the end of the last summer) to depth $z$, as depicted in Fig. 5, and can be written as

$$
\gamma_{V o l}=e^{i k_{z} z_{0}} \frac{\int_{-\infty}^{0} \sigma_{v}(z) e^{i k_{z V o l} z} d z}{\int_{-\infty}^{0} \sigma_{v}(z) d z}
$$

with topographic phase $\mathrm{e}^{i \mathrm{k}_{z} z_{0}} . \quad k_{z V o l}$ is the vertical wavenumber in the subsurface volume, considering refraction and permittivity in the glacier subsurface [16]

$$
k_{z V o l}=\frac{4 \pi \sqrt{\epsilon_{r}}}{\lambda} \frac{\Delta \theta_{r}}{\sin \theta_{r}},
$$

with $\lambda$ being the wavelength in free space and $\epsilon_{r}$ the bulk relative permittivity of the subsurface volume in which the signal penetrates into. $\Delta \theta_{r}$ is the angular difference, after considering refraction into the firn, between the two radar look vectors of the interferograms, driven by the spatial baseline between the acquisitions, as depicted in Fig. 5. Similarly, $\theta_{r}$ is the refracted incidence angle within the firn volume given by Snell's law. The permittivity $\epsilon_{r}$ can be derived from the density

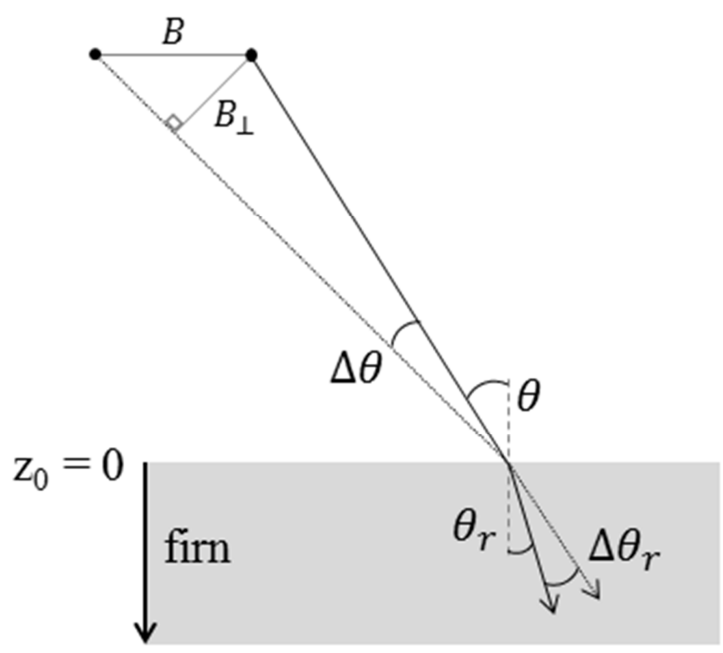

Fig. 5. InSAR geometry considering the refraction into the firn body, ignoring the shallow, partial cover of low density snow.

of firn cores [27] through established relationships [34] and is set to 2.0 throughout this analysis. We use a real valued permittivity since scattering losses dominate over absorption losses under dry and frozen conditions [34].

During the field work, a partial cover of loose snow of about $5 \mathrm{~cm}$ was present on top of an already compacted surface. The last substantial snow height increase measured at the South Dome weather station of the Greenland Climate Network [32] was about $10 \mathrm{~cm}$ around $20^{\text {th }}$ March 2015, 41 and 56 days prior to our SAR acquisitions. This fine grained, low density snow, which is likely to be transparent at microwave frequencies, is ignored here, due to its marginal extent.

Fig. 6 shows the magnitude of the interferometric coherence $|\gamma|$ at L-band in $\mathrm{HH}$ polarization for five different baselines with average $k_{z V o l}$ between $0.19 \mathrm{rad} / \mathrm{m}$ and $0.78 \mathrm{rad} / \mathrm{m}$. In general, coherence values are very low due to strong volume decorrelation, which was expected after the PolSAR analysis. An undulation of coherence is observed in range, which changes with baseline. The analysis of the full data set showed that the undulation pattern changes also with frequency. In fact, the undulation is a function of vertical wavenumber $k_{z V o l}$.

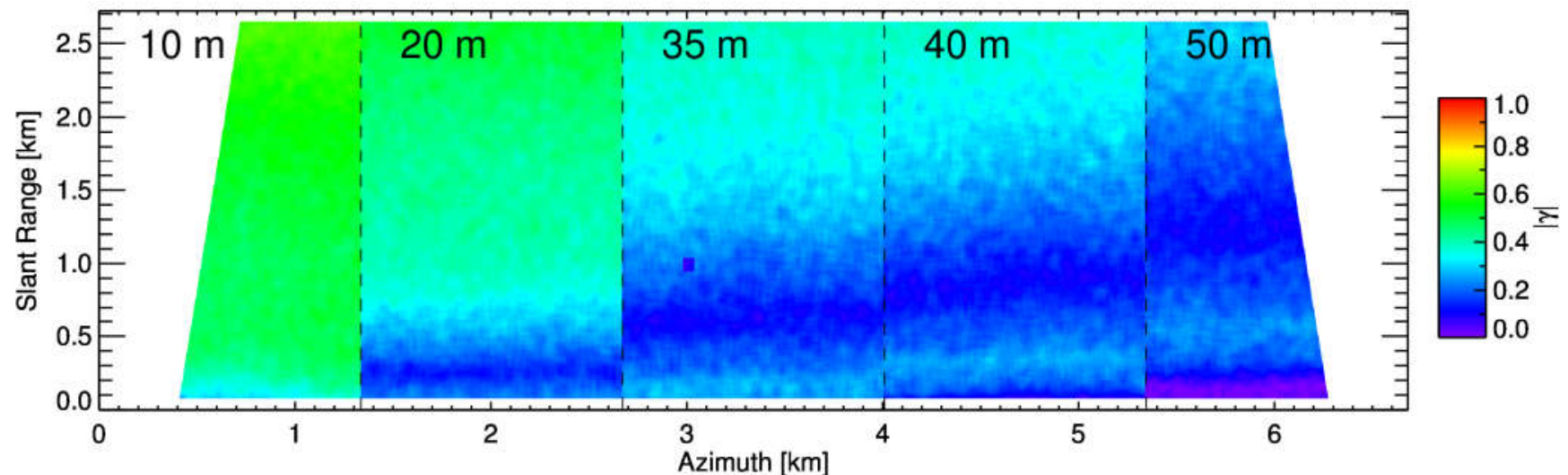

Fig. 6. L-band interferometric HH coherence at five different horizontal baselines, indicated in the image, at a flight altitude of $3000 \mathrm{~m}$ over ground at the South Dome test site. Overall a strong volume decorrelation is observed which is undulated with range in dependence of horizontal baseline. Large estimation windows of at least 3200 looks are applied to reduce coherence estimation bias. Average vertical wavenumbers $k_{z V o l}$ are $0.19,0.30,0.49,0.58$, and $0.78 \mathrm{rad} / \mathrm{m}$, respectively. The effect of a corner reflector is visible in the middle of the scene at $3 \mathrm{~km}$ azimuth and $1 \mathrm{~km}$ slant range. 


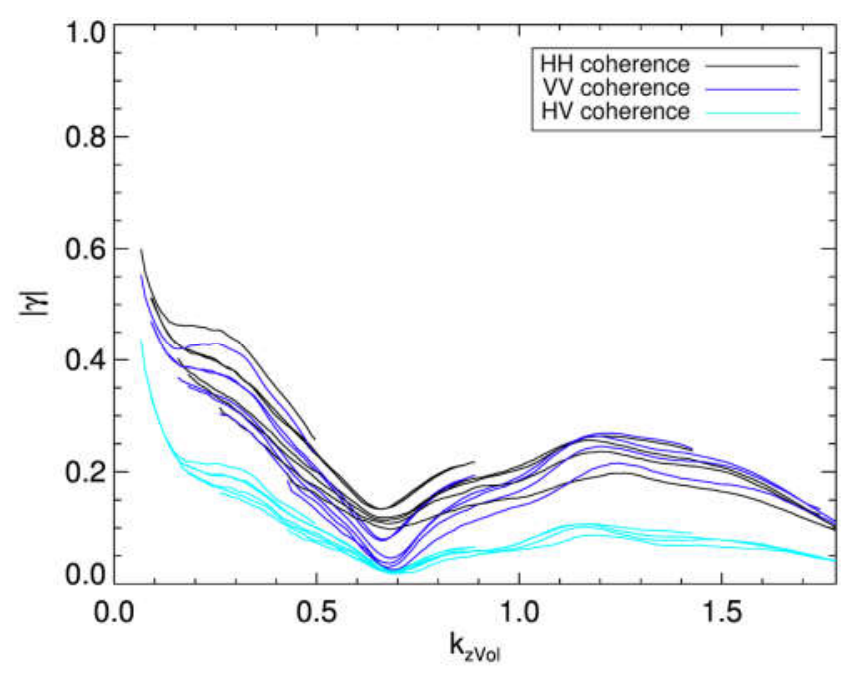

Fig. 7. Coherence profiles at L-band for South Dome vs. $k_{z V o l}$ for six different horizontal baselines (Between $10 \mathrm{~m}$ and $50 \mathrm{~m}$ horizontal baseline, flight altitude $3000 \mathrm{~m}$ above ground). $\mathrm{HH}, \mathrm{VV}$ and $\mathrm{HV}$ profiles are depicted in black, blue and cyan, respectively. The vertical spread at each polarization is related to the fact that the same $k_{z V o l}$ values correspond to different incidence angles for different baselines.

Average L-band coherence profiles are shown in Fig. 7 and demonstrate the dependency on $k_{z V o l}$. We derived the coherence profiles for this analysis using 2D histograms of $|\gamma|$ against $k_{z V o l}$ for each scene. In order to cope with the low $|\gamma|$ values, large estimation windows with at least 3200 looks are used to reduce the coherence estimation bias and standard deviation. The slant range size of the resulting estimation windows is up to $86 \mathrm{~m} \mathrm{x} 181 \mathrm{~m}$, which is no problem due to the horizontal homogeneity of the test site. The profiles from six different horizontal baselines from $10 \mathrm{~m}$ to $50 \mathrm{~m}$ strongly overlap. The coherence profile from the shortest baseline with $0.05 \mathrm{rad} / \mathrm{m}$ (in far range) $<k_{Z V o l}<0.5 \mathrm{rad} / \mathrm{m}$ (in near range) is the only profile not affected by the main undulation pattern, see also Fig. 6 , with a coherence minimum at $k_{z V o l} \approx 0.65 \mathrm{rad} / \mathrm{m}$. It is important to clarify that due to the different horizontal baselines behind each profile, the same $k_{z V o l}$ corresponds to different incidence angles. This leads to different surface scattering contributions at the same $k_{z V o l}$, which is the reason for the vertical variation of the overlapping profiles.

The uniform volume model [11] for InSAR coherences from ice sheets assumes an infinitely deep volume of uniformly distributed scatterers characterized by a constant extinction. It predicts an exponential decay of the coherence with $k_{z V o l}$ (solid line in Fig. 8), which is not observed in the data. The differences between the copol and cross-pol profiles indicate different vertical structure functions for different polarization channels.

We therefore conclude that the shown coherence undulation and the polarization dependency introduce the necessity for a different scattering model for the interpretation of Pol-InSAR data over glaciers and ice sheets.

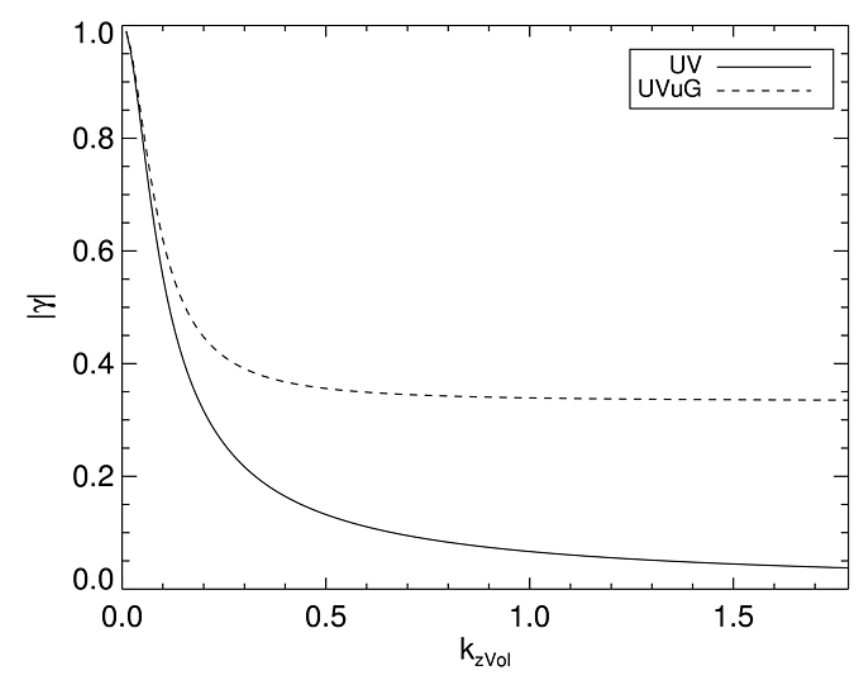

Fig. 8. One example of a coherence profile from a uniform volume model with penetration depth parameter set to $30 \mathrm{~m}$ and a uniform volume under ground model with ground-to-volume ratio 0.5 .

\section{Multiple Layer Plus Volume Model}

In this section, we discuss interferometric coherence models appropriate to interpret the experimental data. The coherence model for a uniform volume, a two layer structure and their combination is introduced step by step. Simulations are used to illustrate the effects of the key parameters layer depth, layer-to-volume scattering ratio and number of layers.

\section{A. Uniform Volume Model}

Assuming a uniform distribution of scatterers with a constant extinction coefficient $\kappa_{e}(\vec{w})$ [11], the vertical structure function $\sigma_{v}(z)$ in (2) becomes an exponential

$$
\sigma_{v}(z)=\sigma_{v}^{0}(\vec{w}) \mathrm{e}^{\frac{2 z \kappa_{e}(\vec{w})}{\cos \theta r}}=\sigma_{v}^{0}(\vec{w}) \mathrm{e}^{\frac{2 z}{d_{p e n}(\vec{w})}},
$$

where $z$ is the vertical (depth) axis, $\cos \theta_{r}$ accounts for the offvertical radar look vector, $\sigma_{v}^{0}(\vec{w})$ is the nominal backscatter power per unit volume and the extinction coefficient $\kappa_{e}(\vec{w})$ accounts for both scattering and absorption losses for a given polarization channel $\vec{w}$. Henceforth, we use the parameterization with one-way penetration depth $d_{p e n}$, which is inversely related to $\kappa_{e}$ through $\kappa_{e}=\cos \left(\theta_{r}\right) / d_{p e n}$. Simulations of $\gamma_{V o l}$ for a uniform volume model lead always to a quasi-exponential coherence decrease (solid line in Fig. 8) and are given by [11]

$$
\gamma_{v o l}=e^{i k_{z} z_{0}} \frac{1}{1+\frac{i d_{p e n}(\vec{w}) k_{z V o l}}{2}} .
$$

Additional scattering contributions from the surface, modelled as a Dirac delta function at depth $0 \mathrm{~m}$, lead to the uniform volume under ground model [19] with one additional parameter, namely the ground-to-volume ratio. This ratio defines the lower coherence limit which is asymptotically reached for larger $k_{z \mathrm{Vol}}$ values (dashed line in Fig. 8). 
In Section II.D, we already discussed why the uniform volume and uniform volume under ground models (Fig. 8) cannot explain our measurements (Fig. 7). Therefore, we extend the uniform volume under ground model in order to account not only for additional scattering from the surface [16], but also scattering from layers within the firn volume.

It is worth highlighting that the uniform volume model is used as a single-channel model without any assumptions on the polarization dependence of the volume scattering. A random volume scenario is obtained assuming the same one-way penetration depth $d_{\text {pen }}$ for all polarizations $\vec{w}$ while an oriented volume scenario is given when $d_{\text {pen }}$ is polarization dependent.

\section{B. Two Layer Model}

In case of a simple vertical structure consisting of two equally strong layers (e.g. the snow-firn interface and one subsurface layer) without any volume contribution, their interference pattern leads to a periodic coherence magnitude undulation with $k_{z V o l}$ (Fig. 9). Neglecting multiple scattering, the interferometric coherence is given by

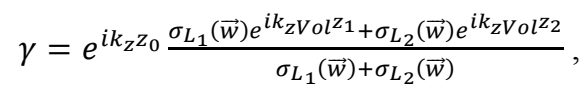

with layer backscattering powers $\sigma_{L_{1,2}}(\vec{w})$ and layer depths $z_{1,2}$.

The minima positions are located at

$$
k_{z V o l}=n \pi /\left(z_{1}-z_{2}\right)
$$

with $n=1,3,5, \ldots$, which is indicated in Fig. 9 by the second $\mathrm{x}$-axis. The periodicity of the coherence minima is similar to the behavior observed not only at L-band (Fig. 7), but also at all other frequencies at the South Dome site. Increasing the vertical distance between the two layers moves the coherence minimum position towards smaller $k_{z V o l}$ values.

While the distance between the layers drives the $k_{Z \mathrm{~V} o l}$ positions of the minima and maxima, it does not affect the extrema values of the coherence magnitude. The coherence magnitude values are sensitive to the scattering powers of the layers $\sigma_{L_{1,2}}(\vec{w})$, which in turn do not affect the $k_{z V o l}$ position of the minima and maxima. According to (7), when the layer distance $z_{1}-z_{2}$ becomes an even multiple of $\pi / k_{z V o l}$, the two layers are in phase and the coherence reaches 1 , independently of the individual $\sigma_{L_{1,2}}(\vec{w})$ values. On the contrary, the coherence magnitude at the minimum depends on the difference in scattering power of the layers. The layers interfere destructively if $z_{1}-z_{2}$ is an odd multiple of $\pi / k_{z V o l}$ and the coherence drops to 0 for $\sigma_{L_{1}}=\sigma_{L_{2}}$. The coherence minimum increases with increasing contrast (i.e. difference in power) between the two layers with $\left|\gamma_{\min }\right|=\left|\sigma_{L_{1}}-\sigma_{L_{2}}\right| /\left(\sigma_{L_{1}}+\sigma_{L_{2}}\right)$. Accordingly, the coherence undulation is the same regardless whether the first or second layer is stronger as long as their difference is the same.

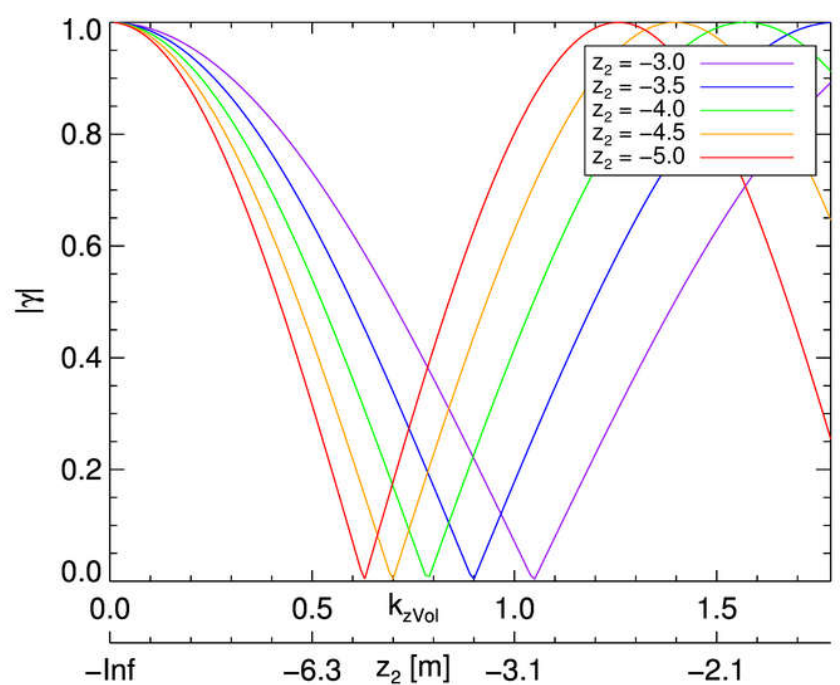

Fig. 9. Coherence magnitude simulations for two layers only with varying depth of the second layer. The first layer is at $z_{1}=0 \mathrm{~m}$ and the depth of the second layer $z_{2}$ is varied between $-3.0 \mathrm{~m}$ and $-5.0 \mathrm{~m}$ as indicated by the colored lines. The scattering powers of the two layers are equal $\sigma_{L_{1}}=\sigma_{L_{2}}=1$. The second $\mathrm{x}$-axis is not a real $\mathrm{x}$-axis, but indicates the depth of the second layer for a given position of the coherence minimum.

\section{Layer plus Volume Model}

In order to explain the overall low coherence values and the steep coherence drop at small $k_{z V o l}$ in Fig. 7, a volume decorrelation contribution needs to be considered additionally. The resulting vertical structure function is then given by the sum of (4) and Dirac delta functions $\delta$ for each of the $N$ layers located at depth $z_{j}$ with layer backscattering power $\sigma_{L_{j}}(\vec{w})$

$$
\sigma_{v}(z)=\sigma_{v}^{0}(\vec{w}) \mathrm{e}^{\frac{2 z}{d_{p e n}(\vec{w})}}+\sum_{j=1}^{N} \sigma_{L_{j}}(\vec{w}) \delta\left(z_{j}\right)
$$

The interferometric coherence is given by

$$
\gamma=e^{i k_{z} z_{0}} \frac{\gamma_{V o l}\left(k_{z V o l}, d_{p e n}(\vec{w})\right)+\sum_{j=1}^{N} m_{j}(\vec{w}) e^{i k_{Z V o l} z_{j}}}{1+\sum_{j=1}^{N} m_{j}(\vec{w})}
$$

with

$$
m_{j}(\vec{w})=\sigma_{L j}(\vec{w}) / \int \sigma_{v}^{0}(\vec{w}) \mathrm{e}^{\frac{2 z}{d p e n(\vec{w})}}
$$
being the layer-to-volume scattering ratio. $\gamma_{V o l}\left(k_{z V o l}, d_{\text {pen }}(\vec{w})\right)$ is the coherence of a uniform volume model with infinite depth, as given in (5), but omitting the topographic phase of (5).

The predictions of such a combined model are shown in Fig. 10 for $N=2$ and $d_{\text {pen }}=30 \mathrm{~m}$. The layer-to-volume ratios $m_{j}$ are fixed to 0.2 for the surface layer and ranges between 0.1 and 0.4 for the second layer located at $-4.5 \mathrm{~m}$ depth. The simulated vertical structure is sketched in the inset. The following points are worth being mentioned:

- The fast drop of coherence magnitude with increasing $k_{z V o l}$ until the first minimum is mainly driven by the decorrelation of the volume component and becomes therefore stronger with increasing volume-only penetration depth. 


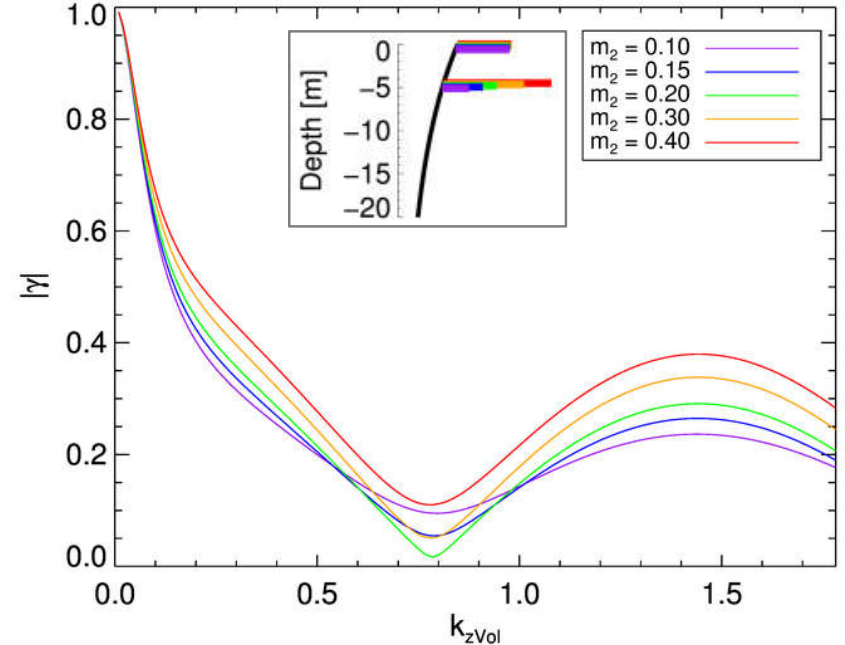

Fig. 10. Two layer plus uniform volume model. The layer-to-volume ratio $m_{1}$ of the first layer located at $z_{1}=0 \mathrm{~m}$ is fixed to 0.2 . The second layer is at $z_{2}=-4.5 m$ with $m_{2}$ varying between 0.1 and 0.4 , as indicated by the colored lines. The volume is modelled by a uniform volume with infinite depth and one-way penetration of $30 \mathrm{~m}$. The simulated vertical structure is sketched in the inset.

- $\quad$ The position of the first coherence minimum is largely determined by the vertical distance of the two layers, according to (7), with a small shift induced by the volume.

- The coherence value at the minimum becomes lowest for $m_{1}=m_{2}$.

- The second coherence maximum $\gamma_{\max 2}$ can be approximated by the sum of the layer-to-volume ratios through

$$
\left|\gamma_{\max 2}\right|=\frac{\sum_{j=1}^{N} m_{j}}{1+\sum_{j=1}^{N} m_{j}}
$$

because $\gamma_{V o l}$ tends to zero for larger $\mathrm{k}_{\mathrm{ZVol}}$ (e.g. $\left|\gamma_{V o l}\right|<0.05$ for $d_{\text {pen }}>28 \mathrm{~m}$ at $k_{z V o l}=1.4 \mathrm{rad} / \mathrm{m}$ ).

Under the assumption that the volume is already completely decorrelated at the first minimum of the undulation pattern, i.e. $\gamma_{V o l}=0+i 0$, we can derive an approximation for the coherence value $|\gamma|$ at this first minimum. The minimum decreases for decreasing layer-to-volume ratio difference $\left|m_{1}-m_{2}\right|$, as described for the two layer only case in Section III.B. Additionally, the coherence value at the minimum is affected by the power of the layers compared to the volume leading to

$$
\left|\gamma_{\min }\right|=\frac{\left|m_{1}-m_{2}\right|}{1+\sum_{j=1}^{N} m_{j}}
$$

To further illustrate the model behavior, Fig. 11 shows the effect of the sum of the layer-to-volume ratios $\sum m_{j}$ for $m_{1}=m_{2}$. The case of $\sum m_{j}=0.4$ (see red line in Fig. 11) corresponds to $m_{2}=0.2$ in Fig. 10 (green line). The effect of the layers, compared to the uniform volume model (purple line in Fig. 11), is clearly visible. The second coherence maximum

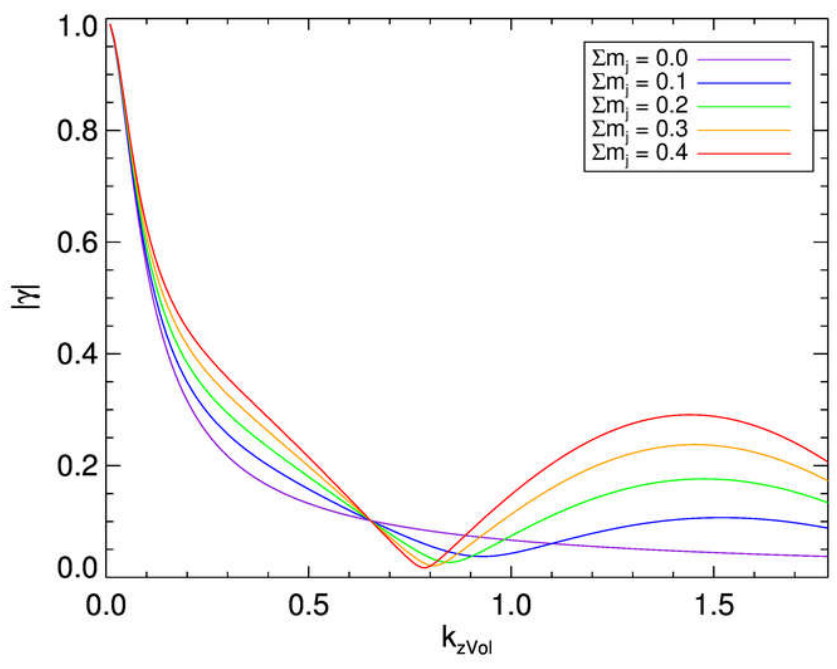

Fig. 11. Effect of the sum of layer-to-volume ratios $\sum m_{j}$ for a two layer plus volume model. Layer parameters are $m_{1}=m_{2} ; z_{1}=0, z_{2}=-4.5 \mathrm{~m}$. The sum of layer-to-volume ratios $\sum m_{j}$ varies between 0.0 and 0.4 . The case of $\sum m_{j}=0.4$ (red line) corresponds to $m_{2}=0.2$ in Fig. 10 (green line).

is mainly driven by $\sum m_{j}$, while the position and the value of the coherence minimum are less sensitive to $\sum m_{j}$-changes above 0.2 . Interestingly, the layers barely affect the coherences for $k_{z V o l}<0.1 \mathrm{rad} / \mathrm{m}$, but their effect is obvious between $k_{z V o l}=$ $0.1 \mathrm{rad} / \mathrm{m}$ and the coherence minimum. The volume influences the coherences starting from $k_{z V o l}=0 \mathrm{rad} / \mathrm{m}$. Accordingly, layers and volume similarly contribute to the coherence in the range $0.1 \mathrm{rad} / \mathrm{m}<k_{z V o l}<0.6 \mathrm{rad} / \mathrm{m}$. At larger $k_{z V o l}$ the effect of the volume is marginal.

The effect of an increasing number of layers needs to be analyzed when thinking about the firn structure at the test site [27] as indicated by the GPR data in Fig. 2. As explained in Section III.B, the superposition of layers leads to undulations, where the periodicity in $k_{z \mathrm{Vol}}$ direction is determined by the distance of the layers while the magnitude of the undulation is driven by the layer-to-volume ratios. Fig. 12 shows the effect of an additional weaker layer $\left(0.02<m_{3}<0.06\right)$ at a depth of $z_{3}=-20.5 \mathrm{~m}$, which is visible in the GPR data in Fig. 2. This simulation roughly matches the higher order undulations of the data in Fig. 7. Thus, an increasing number of layers allows to generate more complex coherence undulation patterns.

Note that even several layers would eventually constructively interfere after the first minimum, leading to higher coherence magnitudes than observed if no volume is considered.

\section{Vertically Extended Subsurface Layers}

Up to now, the scattering layers were considered to be Dirac delta functions at a certain depth $z_{j}$. There are several reasons, why a layer may have a vertical extent. In the percolation zone of Greenland, the formation of refrozen melt-layers is complex and different mechanisms exist that lead to laterally continuous scattering layers [35]. Therefore, there is a chance that these layers are extended over a small depth range. Furthermore, 


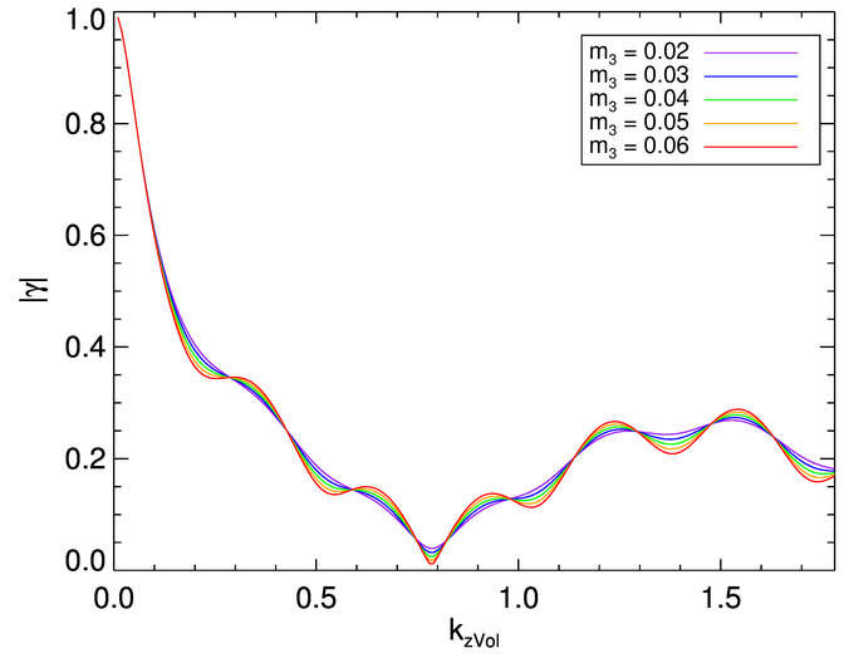

Fig. 12. Three layer plus uniform volume model. The first two layers are fixed at $m_{1}=0.2 ; m_{2}=0.15 ; z_{1}=0 ; z_{2}=-4.5 \mathrm{~m}$. The third layer is located at $z_{3}=-20.5 \mathrm{~m}$ with weaker powers $m_{3}$ varying between 0.02 and 0.06 . The volume is modelled by a uniform volume with infinite depth and one-way penetration of $30 \mathrm{~m}$.

large estimation windows are required to obtain an unbiased coherence estimation at low coherence values, likely associated to small changes in layer depth within their spatial coverage.

We assessed the influence of a layer extent $d_{j}$ for each layer $j$. This leads, for each layer, to a sinc like contribution [36] to the overall coherence, resulting theoretically in a widening of the coherence minima and a lowering of the maxima. However, for the first minimum the widening effect is negligible, even for a $2 \mathrm{~m}$ vertical extent of the layer at $-4.5 \mathrm{~m}$. The same is true for the coherence maxima. We therefore stick to the representation of layers with Dirac delta functions.

\section{Results}

\section{A. Multifrequency Coherence Profiles}

The multiple-layer-plus-volume model is now used to analyze the coherence profiles from the percolation zone of Greenland. Fig. 13 shows the dependency of the coherence magnitude $|\gamma|$ on the vertical wavenumber $k_{z V o l}$ averaged over the entire scene for six interferograms available at each frequency.

At P-band the $\mathrm{HH}$ and VV coherences are almost identical, which implies similar vertical structure functions, while HV has lower coherences and a less pronounced undulation. The second maximum shows the weaker layer contributions in HV as expected, which is true for all frequencies. The small difference between $\mathrm{HH}$ and VV in P-band for low $k_{z V o l}$ values could be an indication of a slightly oriented volume with different $d_{\text {pen }}$ for $\mathrm{HH}$ and $\mathrm{VV}$. This could be caused by polarization dependent scattering in the firn and from ice inclusions which are not part of the layers. The lower coherence in $\mathrm{HV}$ is due to the lower HV layer-to-volume ratios according to our model.

At L-band the minima at $\mathrm{VV}$ are lower than at $\mathrm{HH}$, which means, according to (11), that the $\left|m_{1}-m_{2}\right|$ layer difference is smaller at VV. The second coherence maximum is almost equal in $\mathrm{HH}$ and $\mathrm{VV}$, which implies that the sum of layer-to-volume ratios $\sum m_{j}$ is similar, according to (10). L-band shows also a higher order undulation which may indicate the presence of a third layer.

The S-band profiles show also an undulation, but not as pronounced as at the other frequencies. Also the minimum positions for $\mathrm{HH}$ and $\mathrm{VV}$ seem to be at different $k_{z V o l}$ values. The very wide coherence minima could be explained by vertically extended layers, see Section III.D, but would require unrealistically large vertical distributions of the layer structure functions.

A clear minimum is present at $\mathrm{C}$-band in $\mathrm{HH}$, but is less pronounced in VV. Interestingly, the minima appear at higher $k_{z V o l}$ values than for the P- and L-band frequencies, indicating scattering contributions from a shallower subsurface layer.

Finally, the X-band profiles have a well pronounced coherence undulation with a clearly visible second minimum. $\mathrm{HH}$ and VV maxima are similar, indicating similar sums of layer-to-volume ratios $\sum m_{j}$, but at $\mathrm{VV}$ the difference between the two layers is smaller.

\section{B. Layer Depth Dependence on Frequency}

Using the relationship between layer spacing and minimum position of the coherence profiles in (7), and neglecting a small shift introduced from the volume coherence, we can calculate the depth of the second layer by assuming the first layer to be at $z_{1}=0 \mathrm{~m}$. Looking only at the dominant minima at each frequency in Fig. 13, the second layer is estimated to be roughly at $-3.0 \mathrm{~m}$ at $\mathrm{C}$ - and $\mathrm{X}$-band and $-4.75 \mathrm{~m}$ at $\mathrm{P}$ - and L-band. At $\mathrm{S}-$ band the minima positions are different across polarizations. The VV and HV minimum position is approximately at $k_{z V o l}=$ $0.7 \mathrm{rad} / \mathrm{m}$ which corresponds to $-4.5 \mathrm{~m}$, similar to $\mathrm{L}$ - and P-band. Contrarily, the $\mathrm{HH}$ minimum position is roughly at $k_{z V o l}=1.0 \mathrm{rad} / \mathrm{m}$, which corresponds to a layer depth of $-3.1 \mathrm{~m}$, similar to C- and X-band. Even though it is not clear why the polarizations behave differently at S-band, it seems that S-band is like a transition frequency for the particular vertical subsurface structure at South Dome.

A layer located at $-4.75 \mathrm{~m}$, as indicated by the P- and L-band data, fits well to the dominant layer at $-4.5 \mathrm{~m}$ in the GPR data (Fig. 2). Note that the volume contribution introduces also a small shift in the minimum position, which can lead to an underestimation of layer depth of e.g. $0.5 \mathrm{~m}$ for $d_{\text {pen }}=30 \mathrm{~m}$. This shift becomes smaller with larger $d_{p e n}$.

In case the first scattering layer is not at the surface, i.e. the snow-firn interface, the layers visible at $-4.75 \mathrm{~m}$ and $-10.0 \mathrm{~m}$ in the GPR data, could also explain the coherence undulation at Land P-band, because they have approximately the required vertical distance to produce the observed undulation periodicity.

The higher frequencies, particularly $\mathrm{C}$ - and X-band, indicate a subsurface layer at $-3.0 \mathrm{~m}$, which is not visible in the GPR data. Possible reasons for this are the limited vertical resolution 

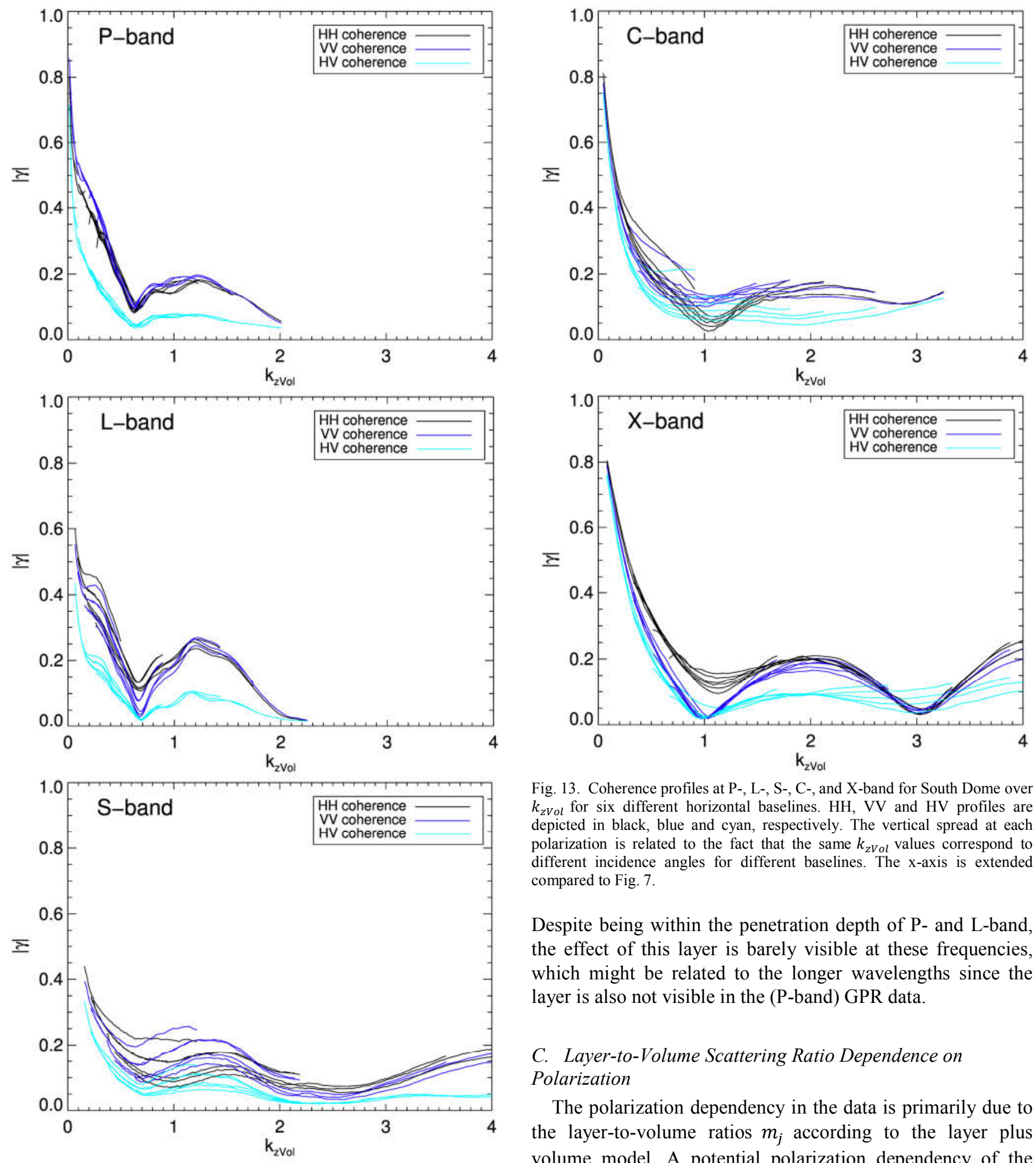

Fig. 13. Coherence profiles at P-, L-, S-, C-, and X-band for South Dome over $k_{z V o l}$ for six different horizontal baselines. HH, VV and HV profiles are depicted in black, blue and cyan, respectively. The vertical spread at each polarization is related to the fact that the same $k_{z V o l}$ values correspond to different incidence angles for different baselines. The $\mathrm{x}$-axis is extended compared to Fig. 7.

Despite being within the penetration depth of P- and L-band, the effect of this layer is barely visible at these frequencies, which might be related to the longer wavelengths since the layer is also not visible in the (P-band) GPR data.

\section{Layer-to-Volume Scattering Ratio Dependence on Polarization}

The polarization dependency in the data is primarily due to the layer-to-volume ratios $m_{j}$ according to the layer plus volume model. A potential polarization dependency of the volume (i.e. an oriented volume) is barely indicated and is discussed in the next Section. Only at S-band, there seems to be an additional polarization sensitivity to the layer depth $z_{j}$ as described above. The simulations in Section III show that there are two key parameters that describe the coherence profiles if only two layers are assumed: First the normalized difference of layer-to-volume scattering ratios $\left|m_{1}-m_{2}\right|$, and second the sum of the layer-to-volume ratios $\sum m_{j}$. use our manual layer probing measurements to better assess near surface layering. The only consistently detected layer was located at $-3 \mathrm{~m}$ depth and was sometimes not even permeable with the probe, see Fig. 3. While all other layers were permeable with one thrust, the layer at $-3 \mathrm{~m}$ had a significant vertical extent. This observation fits to the necessity of a scattering layer at $-3 \mathrm{~m}$ to explain the $\mathrm{C}$ - and $\mathrm{X}$-band profiles. 
TABLE I

SUM OF LAYER-TO-VOLUME SCATTERING RATIOS $\sum m_{j}$

\begin{tabular}{cccc}
\hline$\sum \boldsymbol{m}_{\boldsymbol{j}}$ & $\mathbf{H H}$ & $\mathbf{V V}$ & $\mathbf{H V}$ \\
\hline $\mathbf{P}$ & 0.23 & 0.25 & 0.09 \\
$\mathbf{L}$ & 0.34 & 0.35 & 0.12 \\
$\mathbf{S}$ & 0.17 & 0.22 & 0.10 \\
$\mathbf{C}$ & 0.19 & 0.20 & - \\
$\mathbf{X}$ & 0.25 & 0.22 & 0.10 \\
\hline
\end{tabular}

Average sum of layer-to-volume scattering ratios $\sum m_{j}$ approximated from the coherence profiles in Fig. 13 with (10) (mean values from Fig. 14). For $\mathrm{C}$-band $\mathrm{HV}$ no value is derived because the coherence profiles do not have a clear second coherence maximum.

$\sum m_{j}$ directly affects the second coherence maximum. At these $k_{z V o l}$ values it is fair to assume that the volume coherence is very close to zero. In this case, we can directly infer the sum of layer ratios from the plots, see Table I, through (10). The HV layer scattering ratios are by a factor of 1.7-2.9 lower than at copol channels, while $\mathrm{HH}$ and $\mathrm{VV}$ are very similar. An exception is here again the S-band, where the different polarizations also seem to be sensitive to different layers.

Furthermore, the sum of layer-to-volume ratios $\sum m_{j}$ is derived for different incidence angles, because the second coherence maxima found at a certain $k_{z V o l}$ value correspond to different incidence angles for different horizontal baselines. The incidence angle dependence of $\sum m_{j}$ is shown in Fig. 14 where only coherence profiles with a clear second maximum have been considered.

Additional to the general pattern of stronger layer-to-volume ratios in the copol than in the cross-pol channels, the decrease of layer backscattering with increasing incidence angle is well pronounced. This trend is equally strong for $\mathrm{HH}$ and $\mathrm{VV}$, while there are no changes at HV. Only at P-band, the layer-to-volume ratios appear stable over the whole incidence angle range for all polarizations.

Unfortunately, the layer scattering ratios cannot be directly validated. Nevertheless, the observed differences between the co and cross-pol channels and the trends with incidence angle fit to the expected behavior if layer scattering is considered as a rough surface scattering mechanism and given that volume scattering has marginal sensitivity to incidence angle variations [33].

The behavior of layer scattering ratios with incidence angle is in agreement with the qualitative assessment of scattering mechanisms in Section II.C. Polarimetric entropy and alpha parameters (Fig. 4) indicate the decrease of surface scattering with incidence angle, which leads to an increase of the relative strength of volume scattering.

Another observation is that for the frequencies sensitive to the same layer (i.e. P- and L-band at $-4.75 \mathrm{~m}$; C- and X-band at $-3.0 \mathrm{~m}$ under the assumption of $z_{1}=0 \mathrm{~m}$ ), higher layer-to-volume ratios are retrieved for higher frequencies.

The simulations in Section III.C show that the layer-to-volume ratios do not only directly affect the magnitude of the second coherence maxima, but also the coherences at smaller $k_{z V o l}$, even before the first minimum (Fig. 10 and Fig. 11). This is also reflected by the profiles from shorter baselines in Fig. 13, that have slightly higher coherence magnitudes. For

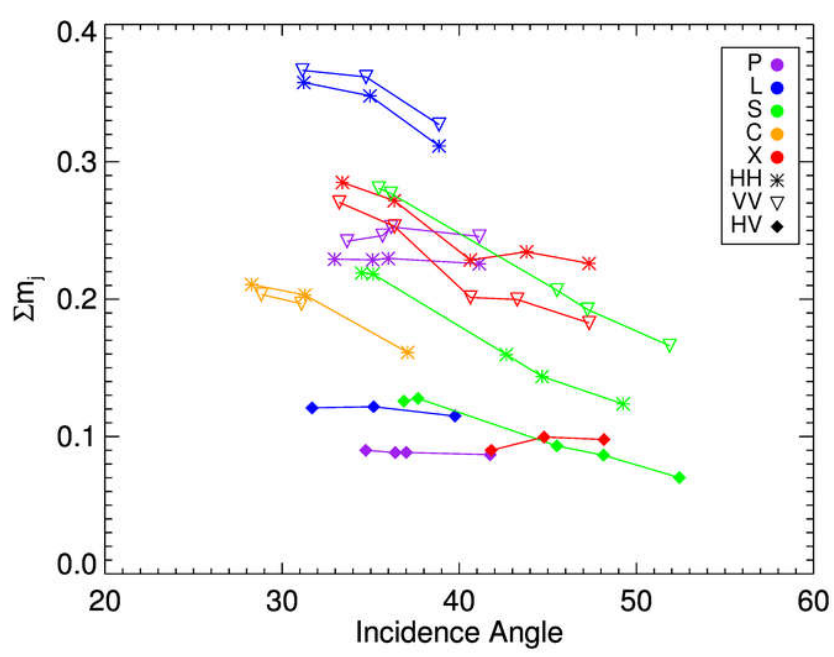

Fig. 14. Sum of layer-to-volume scattering ratios $\sum m_{j}$ derived from the InSAR coherence profiles in Fig. 13 with (10) over incidence angle. The spread across incidence angles comes from the fact that for a given second coherence maximum, the corresponding $k_{Z V o l}$ value is at a different incidence angle for different horizontal baselines. Values are only extracted for profiles with a clear second coherence maximum.

a given $k_{Z V o l}$, these profiles correspond to steeper incidence angles, and therefore to larger layer-to-volume ratios, as shown in Fig. 14. The effect is stronger at L-, S-, and C-band.

\section{Volume Coherence}

The volume contribution is visible in the generally fast drop of coherence values at $k_{z V o l}<0.1 \mathrm{rad} / \mathrm{m}$ in Fig. 13. At L- and $\mathrm{S}$-band this part of the profile cannot be completely presented because of the lack of very small $k_{z V o l}$. At P-, C- and X-band, the available horizontal baselines are small enough to have coherences starting almost at $k_{\text {ZVol }}=0 \mathrm{rad} / \mathrm{m}$.

There is a slight trend of weaker volume decorrelation with increasing frequency confirming less volume penetration at $\mathrm{X}$-band compared to C-band.

At C- and X-band, the coherences are similar across all polarizations for $k_{z V o l}<0.1 \mathrm{rad} / \mathrm{m}$, which could be interpreted as equal $d_{\text {pen }}$ for all polarizations (i.e. random volume) based on the findings from Fig. 11, because the layers have negligible impact for $k_{Z V o l}<0.1 \mathrm{rad} / \mathrm{m}$. The same could be speculated for L- and S-band, but coherences at $k_{z V o l}<0.1 \mathrm{rad} / \mathrm{m}$ are not available. On the contrary, the polarization differences of the coherence profiles at P-band at small $k_{z V o l}$ could indicate an oriented volume with different $d_{\text {pen }}$ for $\mathrm{HH}$ and $\mathrm{VV}$.

\section{E. Model Fit}

From the six baselines at L-band in Fig. 13 we extracted a synthetic average coherence profile and fitted a three layer plus volume model to the data independently for each polarization, as shown in Fig. 15. The possibility for a third layer (i.e. second subsurface layer) was added to account for the higher order undulations in the profile. The first layer was assumed to be at $z_{1}=0 \mathrm{~m}$. The simulations agree well with the general behavior of the L-band coherence profiles, but lack a precise representation of the higher order undulations. 


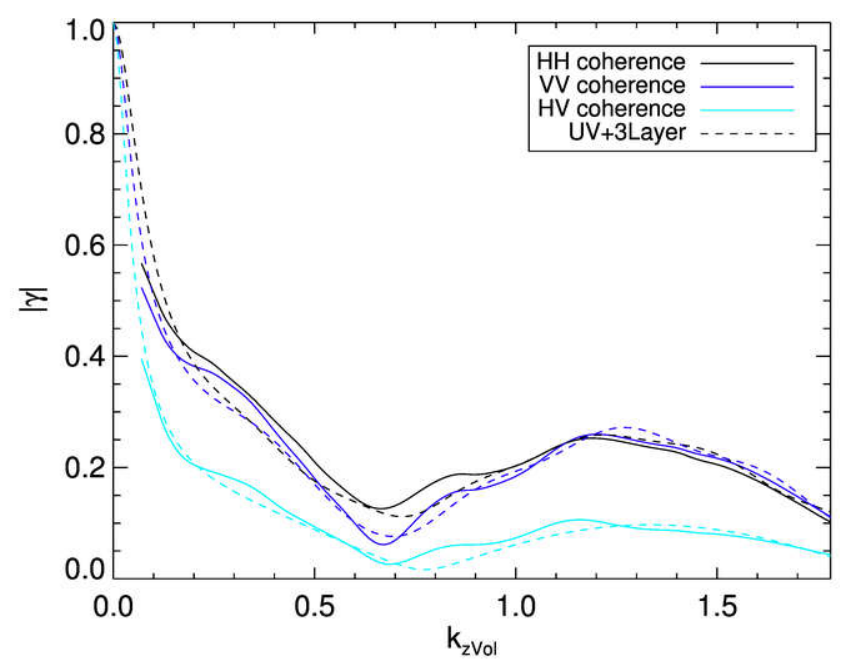

Fig. 15. Three layer plus uniform volume model (dashed lines) fitted to a synthetic mean coherence profile (solid lines) generated for each polarization from six baselines at L-band.

Table II summarizes the parameters obtained by the model fit. The locations of the two subsurface layers at $z_{2}=-5.1 \mathrm{~m}$ and $z_{3}=-21.3 \mathrm{~m}$ agree well, with a mismatch of only $0.1 \mathrm{~m}$ and $1.2 \mathrm{~m}$, respectively, across polarizations, and show only small differences to the layers at $-4.5 \mathrm{~m}$ and $-20.5 \mathrm{~m}$ in the GPR data in Fig. 2. No contribution of a third layer was obtained at HV.

Interestingly, the second layer is stronger than the surface in $\mathrm{VV}$, which leads to a similar undulation pattern as in $\mathrm{HH}$, where the difference of layer-to-volume ratios $m_{1}$ and $m_{2}$ is identical. It is described in Section III.B that the undulation pattern is mainly driven by the layer difference, regardless of which of them is stronger. Layers for HV and the third layer in the copol channels are about one order of magnitude weaker.

The one-way penetration depth values appear very large, but one has to consider that this is the parameter of the volume-only model. The combined penetration depth is shallower due to the layer contributions. The penetration depths differ with polarization, which is an indication for an oriented volume scenario. But the lack of data with $k_{Z V o l}<0.1 \mathrm{rad} / \mathrm{m}$, where the volume contribution can be best assessed, see Section IV.D, hampers a clear assessment of the random vs. oriented volume question. Theoretically, the HV penetration depth has to be in between the values for $\mathrm{HH}$ and $\mathrm{VV}$ in an oriented volume scenario, which is not the case for the results of the model fit. This could indicate a different vertical distribution of backscattered power of the volume at $\mathrm{HV}$, which cannot be interpreted by a uniform volume model.

\section{DISCUSSION AND CONCLUSION}

In this paper, a scattering model for Pol-InSAR coherences from the percolation zone in Greenland has been proposed and validated. We observed a coherence undulation with vertical wavenumber $k_{z V o l}$ in our data that cannot be explained with existing uniform volume only [11] or volume under ground [19] models. For this, the presence of subsurface layers is required. Simulations show how the layered vertical structure
TABLE II

PARAMETERS OF THE THREE LAYERS PLUS UNIFORM VOLUME MODEL FITTED TO THE SYNTHETIC AVERAGE COHERENCE PROFILE AT L-BAND.

\begin{tabular}{cccc}
\hline & HH & $\mathbf{V V}$ & $\mathbf{H V}$ \\
\hline $\mathbf{z}_{\mathbf{1}}[\mathbf{m}]$ & 0 & 0 & 0 \\
$\mathbf{z}_{2}[\mathbf{m}]$ & -5.1 & -5.1 & -5.0 \\
$\mathbf{z}_{\mathbf{3}}[\mathbf{m}]$ & -21.3 & -20.1 & - \\
$\mathbf{m}_{\mathbf{1}}$ & 0.23 & 0.11 & 0.05 \\
$\mathbf{m}_{\mathbf{2}}$ & 0.10 & 0.24 & 0.05 \\
$\mathbf{m}_{\mathbf{3}}$ & 0.007 & 0.015 & 0.0 \\
pen. depth $[\mathbf{m}]$ & 32 & 45 & 60 \\
\hline
\end{tabular}

below the surface affects the InSAR coherences due to the constructive and destructive interference of the layer contributions leading to pronounced coherence minima and maxima in dependence of $k_{z \mathrm{Vol}}$. At all frequencies, two layers are enough to interpret the main coherence magnitude behavior. Only at L-band, a higher order undulation is present that can be described by a third, deeper layer.

Furthermore, the simulations also reveal the potential to estimate and characterize subsurface layers from InSAR coherences. Under the assumption of the first layer being located at the surface, we can interpret the data, by comparison with forward simulations, with a second layer at $-4.75 \mathrm{~m}$ at $\mathrm{P}$ and L-band and at $-3 \mathrm{~m}$ for $\mathrm{C}$ - and X-band, which is supported by in situ data. A model fit to the L-band data indicates a second layer at $-5.1 \mathrm{~m}$, which is considered a good agreement given that the model fit shows small deviations from the data. The situation at S-band is less clear and seems to be a transition between the other frequencies.

Also layer-to-volume scattering ratios can be inferred from the measured coherences, potentially revealing the relative importance of different scattering mechanisms. As expected, cross-pol layer-to-volume scattering ratios are smaller than at copol channels throughout the data set, but a subsurface layer is still necessary to explain the undulations at HV. The VV layer scattering ratios are slightly larger than at $\mathrm{HH}$ for most frequencies, but the differences can be as small as $3 \%$.

The validation is mainly limited to layer depths, which can be inferred from our in situ measurements, since manually measured layer thickness or GPR signals cannot directly be used to validate layer-to-volume ratios based on SAR data. Furthermore, it is not yet completely understood which layers detected in a GPR profile are dominant enough to have an impact on InSAR coherences.

We generally conclude that ice sheet areas exist where the assumptions of a uniform volume or uniform volume under ground scenario are not valid. However, the overall low coherence magnitudes and the retrieved layer-to-volume scattering ratios demonstrate the overall dominance of volume scattering, which is supported by polarimetric analyses and is also consistent with previous studies [11][16]. Despite that, we show clear evidence that dominant subsurface layers have to be taken into account.

The inversion of the proposed model has not been addressed here as it goes beyond the scope of this work, but will strongly depend on the observation scenario as well as on the subsurface characteristics of the area under investigation. The simplest case is a two layer plus volume model, where the first layer is 
assumed to be at the surface and the volume is independent of the polarization (i.e. random volume). The resulting nine model parameters could be theoretically inverted with a dual-baseline Pol-InSAR setup providing twelve independent observables. There is potential in combining observations at small $k_{z V o l}$, which are mainly sensitive to the volume, and at larger $k_{z V o l}$, which are mainly sensitive to the layer contributions. Such an inversion scheme remains to be established. Any additional layer or a polarization dependent volume (i.e. oriented volume) add at least two parameters and will complicate the inversion. For comparison, single-pol single-baseline data allows only the inversion of a uniform volume model without any layers. Full-pol single-baseline data improves the inversion capability to e.g. a random volume under ground model, but the existence of a second layer requires dual-baseline data for any inversion, as described above.

In the percolation zone of Greenland, distinct subsurface layers are related to refreezing of melt water. Therefore, the analysis of coherence patterns could support the assessment of accumulation rates, density changes or melt-refreeze processes. Starting from a clear melt layer structure as we observed at South Dome, the effect of such dominant layers within the firn body is expected to differ for areas with less or no melting during summer towards the dry snow zone. The explicit modelling of depth hoar layers within firn in the dry snow zone of Greenland by Oveisgharan and Zebker [13] relied on a priori information and was compared to accumulation rates from firn cores. Unfortunately, it was impossible to acquire data in the dry snow zone during the ARCTIC15 campaign to see if we could show an effect of a layered subsurface structure directly on a data level as we did for the percolation zone. Additionally, in the other direction, with increasing melt periods, refrozen ice inclusions within the firn can become so abundant that no distinct layers could be present and the vertical structure could be interpreted again with volume scattering models. Therefore, one model seems not to be enough for the interpretation of Pol-InSAR data over different glacier zones of ice sheets, an approach which was already followed by [10] for PolSAR.

The potential to retrieve geophysical information about dominant subsurface layers was validated with in situ data, indicating that Pol-InSAR data could be exploited in the future for space borne retrieval of ice sheet subsurface structure. Further studies are necessary to investigate how a retrieval with a limited number of acquisitions can be achieved.

\section{ACKNOWLEDGMENT}

The authors would like to thank everyone involved in the ARCTIC15 campaign, which was conducted by DLR and ETH Zurich in cooperation with the Danish Defence Acquisition and Logistics Organization (DALO), and the Alfred-Wegener-Institute for providing the GPR system and general support for the campaign.

The authors are grateful for the valuable and constructive comments from the anonymous reviewers, which improved both clarity and quality of the paper.

\section{REFERENCES}

[1] D.G. Vaughan, J.C. Comiso, I. Allison, J. Carrasco, G. Kaser, R. Kwok, P. Mote, T. Murray, F. Paul, J. Ren, E. Rignot, O. Solomina, K. Steffen, and T. Zhang, "Observations: Cryosphere," in Climate Change 2013: The Physical Science Basis. Contribution of Working Group I to the Fifth Assessment Report of the Intergovernmental Panel on Climate Change, [Stocker, T.F., D. Qin, G.-K. Plattner, M. Tignor, S.K. Allen, J. Boschung, A. Nauels, Y. Xia, V. Bex and P.M. Midgley (eds.)]. Cambridge University Press, Cambridge, United Kingdom and New York, USA, 2013.

[2] A. S. Gardner, G. Moholdt, J. G. Cogley, B. Wouters, A. A. Arendt, J. Wahr, E. Berthier, R. Hock, W. T. Pfeffer, G. Kaser, S. R. M. Ligtenberg, T. Bolch, M. J. Sharp, J. O. Hagen, M. R. van den Broeke, F. Paul, “A reconciled estimate of glacier contributions to sea level rise: 2003 to 2009," Science, vol. 340, pp. 852-857, May 2013.

[3] R. Forster, K. C. Jezek, J. Bolzan, F. Baumgartner, and S. P. Gogineni, "Relationships between radar backscatter and accumulation rates on the Greenland ice sheet," Int. J. Remote Sens., vol. 20, no. 15/16, pp. 31313147, Jan. 1999.

[4] G. Rotschky, W. Rack, W. Dierking, and H. Oerter, "Retrieving snowpack properties and accumulation estimates from a combination of SAR and scatterometer measurements," IEEE Trans. Geosci. Remote Sens., vol. 44, no. 4, pp. 943-956, Apr. 2006.

[5] M. A. Fahnestock, R. Bindschadler, R. Kwok, and K. C. Jezek, "Greenland ice sheet surface properties and ice dynamics from ERS-1 SAR imagery," Science, vol. 262, no. 5139, pp. 1530-1534, Dec. 1993.

[6] K. C. Jezek, M. R. Drinkwater, J. P. Crawford, R. Bindschadler, and R. Kwok, "Analysis of synthetic aperture radar data collected over the southwestern Greenland ice sheet," J. Glaciol., vol. 39, no. 131, pp. 119132, Jan. 1993.

[7] E. J. Rignot, S. Ostro, J. van Zyl, and K. Jezek, "Unusual radar echoes from the Greenland ice sheet," Science, vol. 261, no. 5129, pp. 17101713, Sep. 1993.

[8] E. J. Rignot, "Backscatter model for the unusual radar echoes from the Greenland ice sheet," J. Geophys. Res., vol. 100, no. E5, pp. 9389-9400, May 1995.

[9] J. J. Sharma, I. Hajnsek, K. P. Papathanassiou, and A. Moreira, "Polarimetric decomposition over glacier ice with long-wavelength PolSAR," IEEE Trans. Geosci. Remote Sens., vol. 49, no. 1, pp. 519-535, Jan. 2011.

[10] G. Parrella, I. Hajnsek and K. P. Papathanassiou, "Polarimetric Decomposition of L-Band PolSAR Backscattering Over the Austfonna Ice Cap," IEEE Trans. Geosci. Remote Sens., vol. 54, no. 3, pp. 1267-1281, Mar. 2016.

[11] E. W. Hoen and H. Zebker, "Penetration depths inferred from interferometric volume decorrelation observed over the Greenland ice sheet," IEEE Trans. Geosci. Remote Sens., vol. 38, no. 6, pp. 2572-2583, Nov. 2000.

[12] E. Rignot, K. Echelmeyer, and W. Krabill, "Penetration depth of interferometric synthetic aperture radar signals in snow and ice," Geophys. Res. Lett., vol. 28, no. 18, pp. 3501-3504, Sep. 2001.

[13] S. Oveisgharan and H. Zebker, "Estimating snow accumulation from InSAR correlation observations," IEEE Trans. Geosci. Remote Sens., vol. 45, no. 1, pp. 10-20, Jan. 2007.

[14] P. Rizzoli, M. Martone, H. Rott, and A. Moreira, "Characterization of Snow Facies on the Greenland Ice Sheet Observed by TanDEM-X Interferometric SAR Data," Remote Sensing, vol. 9, no. 4, Mar. 2017.

[15] S.R. Cloude and K.P. Papathanassiou, "Polarimetric SAR interferometry," IEEE Trans. Geosci. Remote Sens., vol. 36, no. 5, pp. 1551-1565, Sept. 1998.

[16] J.J. Sharma, I. Hajnsek, and K.P. Papathanassiou, "Estimation of glacier ice extinction using long-wavelength airborne Pol-InSAR," IEEE Trans. Geosci. Remote Sens., vol. 51, no. 6, pp. 3715-3732, Jun. 2013.

[17] O. Stebler, A. Schwerzmann, M. Luthi, E. Meier, and D. Nuesch, "Pol-InSAR observations from an Alpine glacier in the cold infiltration zone at L- and P-band," IEEE Geosci. Remote Sens. Lett., vol. 2, no. 3, pp. 357-361, Jul. 2005.

[18] T. Nagler, H. Rott, I. Hajnsek, K.P. Papathanassiou, and R. Scheiber, “An airborne experiment on snow parameter retrieval by means of multi-channel SAR data," in Proc. EUSAR, Dresden, Germany, May 16-18, 2006

[19] J. Dall, K.P. Papathanassiou, and H. Skriver, "Polarimetric SAR interferometry applied to land ice: Modeling," in Proc. EUSAR, Ulm, Germany, 2004, pp. 247-250. 
[20] S. Leinss, G. Parrella, and I. Hajnsek, "Snow height determination by polarimetric phase differences in X-band SAR data," IEEE J. Sel. Topics Appl. Earth Observ., vol. 7, no. 9, pp. 3794-3810, Jun. 2014.

[21] K. Sainath and S. Hensley, "Numerical modeling of subsurface layer resonance-based interferometric SAR (InSAR) correlation fluctuations," in Proc. EUSAR, Hamburg, Germany, Jun. 6-9, 2016, pp. 567-572.

[22] G. Fischer, G. Parrella, K. P. Papathanassiou, and I. Hajnsek, "Interpretation of Pol-InSAR signatures from glaciers and ice sheets at different frequencies," in Proc. EUSAR, Hamburg, Germany, Jun. 6-9, 2016, pp. 806-811.

[23] E. W. Hoen, "A correlation-based approach to modeling interferometric radar observations of the Greenland ice sheet," Ph.D. dissertation, Stanford Univ., Stanford, CA, Mar. 2001.

[24] R. Horn, A. Nottensteiner, and R. Scheiber, "F-SAR - DLR's advanced airborne SAR system onboard DO228," in Proc. EUSAR, Friedrichshafen, Germany, Jun. 2-5, 2008, pp. 195-198.

[25] I. Hajnsek, K. Papathanassiou, and S. R. Cloude, "Removal of additive noise in polarimetric eigenvalues processing," in Proc. IGARSS, Sydney, Australia, 2001, pp. 2778-2780.

[26] A. Reigber, "Range dependent spectral filtering to minimize the baseline decorrelation in airborne SAR interferometry", in Proc. IGARSS, Hamburg, Germany, 1999, pp. 1721-1723.

[27] J. Freitag, S. Kipfstuhl, S. Hoerz, L. Eling, B. Vinther, and T. Popp, "Melt layer statistic of two firn cores recently drilled at Dye3 and South Dome in the dry snow zone of southern Greenland", presented at EGU General Assembly, Vienna, Austria, Apr. 27-May 2, 2014.

[28] H. M. Jol, Ground Penetrating Radar: Theory and Applications, Amsterdam, The Netherlands: Elsevier, 2009

[29] C. Leuschen, "IceBridge Accumulation Radar L1B Geolocated Radar Echo Strength Profiles, Version 2," subset 8 April 2014 05_016, Nat Snow Ice Data Center, Boulder, CO, 2015.

[30] S.L. Buchardt, H.B. Clausen, B. M. Vinther, and D. Dahl-Jensen, "Investigating the past and recent d18O-accumulation relationship seen in Greenland ice cores," Clim. Past, pp. 2053-2059, Dec. 2012.

[31] C. Leuschen, "IceBridge Snow Radar L1B Geolocated Radar Echo Strength Profiles, Version 2," subset 10 April 2015 01_116, Nat. Snow Ice Data Center, Boulder, CO, 2016.

[32] K. Steffen, J. E. Box, and W. Abdalati, "Greenland Climate Network: GC-Net," in CRREL 96-27 Special Report on Glaciers, Ice Sheets and Volcanoes, trib. to M. Meier, S. C. Colbeck, Ed., pp. 98-103, 1996.

[33] S. R. Cloude, Polarisation: Applications in Remote Sensing, New York: Oxford Univ. Press, 2010.

[34] C. Mätzler, "Applications of the interaction of microwaves with the natural snow cover," Remote Sensing Rev., vol. 2, pp. 259-387, 1987.

[35] T. Dunse, O. Eisen, V. Helm, W. Rack, D. Steinhage, and V. Parry, "Characteristics and small-scale variability of GPR signals and their relation to snow accumulation in Greenland's percolation zone," $J$. Glaciol., vol. 54, no. 185, pp. 333-342, Mar. 2008

[36] S. R. Cloude and K. P. Papathanassiou, "Three-stage inversion process for polarimetric SAR interferometry," IEE Proc - Radar Sonar Navigat., vol. 150, no. 3, pp. 125-134, Jun. 2003. 